\begin{tabular}{|c|c|c|c|}
\hline Eiszeitalter und Gegenwart & 53 & $\begin{array}{c}39-54 \\
7 \mathrm{Abb} .\end{array}$ & Hannover 2003 \\
\hline
\end{tabular}

\title{
Glazialtektonik weichselzeitlicher Ablagerungen in Zentral-Fünen, Dänemark
}

\author{
Jan A. Piotrowski \& Sophia Windelberg ${ }^{*)}$
}

Keywords: glaciotectonics, glaciation, Baltic ice stream, till, Pleistocene, Funen, Denmark

Kurzfassung: Die glazialen Sedimente des Untersuchungsgebietes im zentralen Teil der dänischen Insel Fünen sind durch intensive Deformation geprägt. In den mächtigen glazifluviatilen Sedimentfolgen treten vor allem Überschiebungen, stark deformierte Faltenstrukturen und Zungenfalten auf. Da die Einmessung dieser Strukturen bis zu drei Deformationsrichtungen ergibt, müssen verschiedene Eisbewegungsrichtungen auf die Sedimente eingewirkt haben. Mit Hilfe sedimentologischer Untersuchungen des im Hangenden aufgeschlossenen Tills ist die Bildung eines großen Teils der Deformationsstrukturen mit dem Hauptvorstoß der Weichselzeit aus Nordosten und dem darauffolgenden jungbaltischen Vorstoß aus südöstlicher Richtung zu korrelieren. Die nahezu gleichmäßige Verteilung der verschiedenen Deformationsrichtungen lässt sich auf unterschiedliche Scherfestigkeiten der einzelnen Profilteile zurückführen, die z.T. eine Überprägung der bereits vorhandenen Deformationsstrukturen verhinderten. Es gibt keinen Zusammenhang zwischen der Eisvorstoßdynamik und bestimmten glazialtektonischen Strukturen, deren Stil vielmehr durch rheologische Eigenschaften des Sedimentes bestimmt ist. Die hohe Geschwindigkeit des jungbaltischen, als Eisstrom ausgebildeten Vorstoßes war z.T. durch Verformung des weichen, wasserübersättigten Untergrundes begünstigt.

\footnotetext{
* Anschriften der Verfasser: Prof. Dr. Jan A. PıOTROWSKI, Department of Earth Sciences, University of Aarhus, C.F. Møllers Allé 120, DK-8000 Århus C, Dänemark, jan.piotrowski@geo.au.dk, Dipl.Geol. Sophia WindelberG, Fachbereich Geowissenschaften, Universität Bremen, Klagenfurter Str., 28334 Bremen.
}

\author{
[Glaciotectonics in the Weichselian
} deposits on central Funen, Denmark]

Abstract: The glacial deposits in the study area in the central part of the Danish island Funen are heavily deformed. Thick outwash sediments are characterized by thrust faults, contorted fold structures and sheath folds. Three main deformation vectors indicate different ice movement directions. Coupled with sedimentary studies of the overlying till, these deformations are attributed to the Main Advance of the Weichselian glaciation from NE and the subsequent Young Baltic advance from SE. The mixed distribution of deformation directions throughout the sections is attributed to different shear strength of the deposits which partly prevented overprinting of older structures during younger ice overriding. There is no apparent correlation between ice movement dynamics and particular glaciotectonic structures, whose style was primarily influenced by the sediment rheological properties, instead. The high movement velocity of the Young Baltic ice stream was partly accomplished through the deformation of the soft, water-saturated bed.

\section{Einleitung}

Glazialtektonische Strukturen entstehen in weichem Sediment im Zusammenhang mit Gletscherbewegungen und Auflastdruck. Sie bestehen im Wesentlichen aus Auf- und Überschiebungen, listrisch geprägten Störungen und Faltenstrukturen, die aufgrund ihrer Richtung und ihres Auftretens Hinweise auf die Gletscherbewegungsrichtung und z.T. auf ihre Bildungsposition im Verhältnis zur Lage des 
Gletschers liefern (ABER et al. 1989, VAN DER WATEREN 2002).

Im Untersuchungsgebiet auf der dänischen Insel Fünen begann die Dokumentation glazialer Sedimente und die Korrelation dieser Ablagerungen mit bestimmten Vereisungsphasen bereits 1897 mit Kartierungen von MAdSEN (1902). Die zunächst auf die Entstehung unterschiedlicher Landschaftselemente im Zusammenhang mit Eisvorstößen ausgerichtete Untersuchung wurde Mitte des letzten Jahrhunderts von V. Milthers (1928), K. Milthers (1942) und später von Smed $(1962,1978)$ fortgesetzt. Die heute üblichen sedimentologischen Untersuchungsverfahren, bestehend aus KorngrößenGefüge- und Feinkiesanalysen, wurden auf Fünen von Houmark-Nisisen (1981, 1987 , 1999) im Rahmen von Arbeiten mit stratigraphischem Schwerpunkt durchgeführt. Die Methoden ermöglichen, unabhängig von der Morphologie auf die Bewegungsrichtung und Herkunft des Eises, welches zur Ablagerung und Deformation der Sedimente führte, zu schließen. Der östliche Teil Fünens, in welchem sich das Untersuchungsgebiet der vorliegenden Arbeit befindet, wurde auf diese Art von JørGENSEN (1996) ebenfalls unter stratigraphischen Gesichtspunkten untersucht. Subglaziale Prozesse, insbesondere die Eisbewegungsmechanik und die Entstehung der Drumlins auf Fünen wurden zuletzt von Jørgensen \& PiotrowsKi (2003) behandelt.

Die vorliegende Arbeit verfolgt das Ziel, die Entwicklung einer Abfolge glazialer Sedimente nach ihrer Ablagerung mittels der darin auftretenden Deformationsstrukturen festzustellen. Zu diesem Zweck werden die Richtungen dieser Strukturen in hauptsächlich glazifluviatilen Sedimenten in zwei Profilen untersucht. In Kombination mit sedimentologischen Untersuchungen an den Tills im oberen Bereich der Profilfolge und den auf Fünen dokumentierten Eisvorstoßrichtungen kann die zeitliche und räumliche Entwicklung der Deformation nachvollzogen werden.

\section{Das Untersuchungsgebiet}

Das Untersuchungsgebiet befindet sich auf der dänischen Insel Fünen etwa $13 \mathrm{~km}$ südöstlich der Stadt Odense innerhalb eines ca. 900 ha großen Sand- und Kiesabbaugebietes (Abb. 1 und 2). Im nordwestlichen Teil dieses Areals in der Umgebung der Ortschaft Birkum wurden Profilaufnahmen in zwei nah beieinander gelegenen Kiesgruben durchgeführt. Ein 168 m langes Profil wurde in einer Grube der Firma NCC aufgenommen, welche direkt am nördlichen Rand des Hügels Ibjerg, etwa 1,5 km nordwestlich von Tarup und etwa $1 \mathrm{~km}$ nordöstlich von Sønder Nærå, an der Strasse Ibjergvej liegt. Die Lokalität wird hier kurz als Ibjerg bezeichnet. Ein zweites, $78 \mathrm{~m}$ langes Profil wurde in einer Grube der Firma NS Nymølle Stenindustrier A/S 1,2 km östlich von Birkum aufgenommen. Diese Grube liegt direkt südlich der Straße 301, dem Orbækvej, zwischen Birkum und Rolsted und wird im Folgenden Birkum genannt. Die zwei bearbeiteten Profilstöße liegen ca. $1,5 \mathrm{~km}$ voneinander entfernt. Das Untersuchungsgebiet ist in der direkten Umgebung der Kiesgruben zwischen Davinde im Nordosten und dem Ibjerg im Süden durch eine flachkuppige Moränenfläche (SмгD 1962) geprägt, welche sich nach Westen mehrere Kilometer über das Sand- und Kiesgrubengebiet hinaus ausdehnt und hauptsächlich aus Till besteht. Sie liegt in der Umgebung der Lokalität Ibjerg auf einer Höhe von $48 \mathrm{~m}$ a.s.l., fällt von hier langsam nach Norden und Nordosten ab und befindet sich nahe der Lokalität Birkum auf einer Höhe von $43 \mathrm{~m}$ a.s.l.. Abgesehen von diesem generellen Trend wird sie von einzelnen spät- bis postglazial entstandenen flachen Tälern durchzogen (Jørgensen 1996). Innerhalb des Untersuchungsgebietes bildet der Ibjerg mit einer Höhe von $61 \mathrm{~m}$ a.s.l. die größte Erhebung. Der niedrigste Punkt liegt in einer Depression südöstlich der Ortschaft Birkum bei etwa $39 \mathrm{~m}$ a. s. 1 . (Geodatisk Institut 1987). 


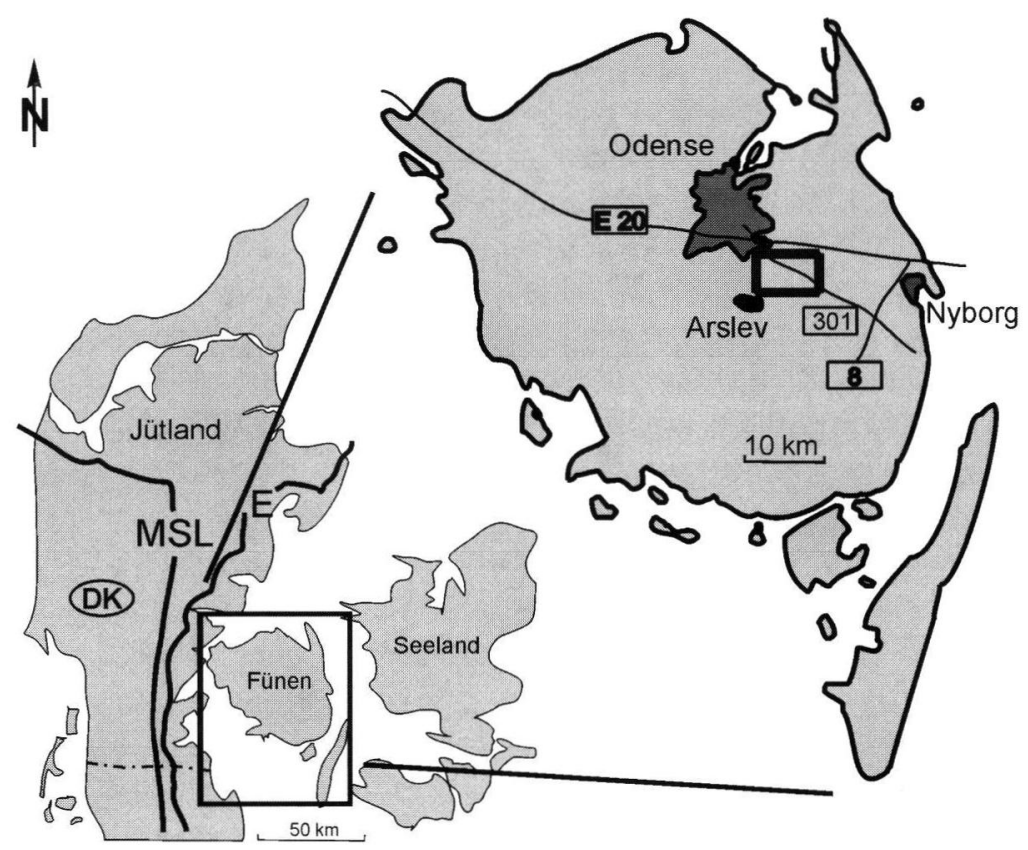

Lage des Untersuchungsgebietes (s. Abb. 2)

- Eisrandlinien weichselzeitlicher Eisvorstöße

MSL Hauptstillstandslinie, Randlage des Hauptvorstoßes der Weichselzeit

E Ostjütische Eisrandlage, Randlage des jungbaltischen Vorstoßes

Abb. 1: Lage des Untersuchungsgebietes auf Fünen und die Eisrandlinien des Haupt- und des jungbaltischen Vorstoßes der Weichseleiszeit in Dänemark.

Fig. 1: The study area on Funen and the limits of the Main and Young Baltic advances in Denmark.

Die Moränenfläche wird im Norden und Osten durch 6 bis $8 \mathrm{~m}$ hohe, in SE-NW Richtung orientierte Drumlins begrenzt. Sie sind Teil eines Drumlinfeldes, welches sich über den gesamten nordöstlichen und nördlichen Bereich der Insel ausdehnt. Die Entstehung dieses Feldes ist auf einen Eisstrom zurückzuführen, der das Gebiet während des Weichsel-Spätglazials bedeckte und aus dem skandinavischen Eisschild über die Senke der Ostsee gespeist wurde (Jørgensen \& Piotrowski 2003, KJÆR et al. 2003). Neben den Drumlins treten in der unmittelbaren Umgebung des Untersuchungsgebietes zahlreiche kleinere Oser auf, welche sich z.T. zu großen Osersystemen verbinden lassen (JøRGENSEN 1996).
Die morphologischen Landschaftselemente des Untersuchungsgebietes und die aufgeschlossenen Sedimentfolgen entstanden während des Jütland-Stadials des WeichselSpätglazials. Aus diesem Stadial sind im Wesentlichen zwei Eisvorstöße bekannt, die vor etwa 20000 Jahren (Petersen \& Kronborg 1991) in Dänemark mit dem sog. Hauptvorstoß einsetzten. Der Gletscher dieses Vorstoßes breitete sich von Nordosten über die dänischen Inseln bis an die als morphologische Struktur gut erkennbare Hauptstillstandslinie (Abb. 1) in Jütland aus und hinterließen im Untersuchungsgebiet einen Till, der meist durch ein ENE-WSW orientiertes Gefüge und einen im Vergleich zu den jüngeren Tills geringen Tonanteil gekenn- 


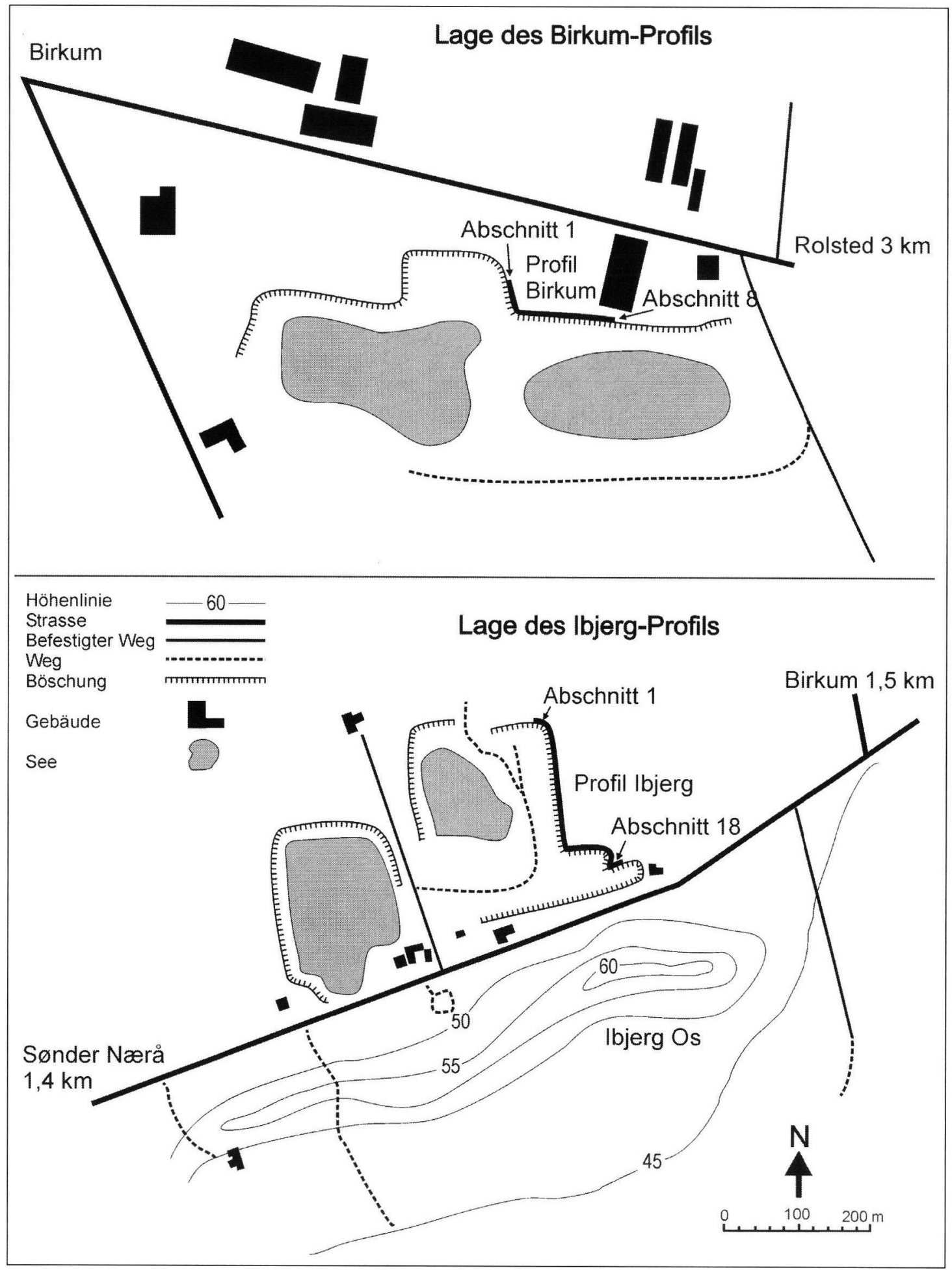

Abb. 2: Beide Lokalitäten im Untersuchungsbebiet und die Lage der Profilverläufe aus Abb. 3 und 6. Fig. 2: The two study sites and the position of sections from Fig. 3 and 6. 
zeichnet ist (JørGENSEN \& PiotrowsKi 2003). Nach dem Abschmelzen der Gletscher des Hauptvorstoßes folgte vor etwa 14000 Jahren (Petersen \& Kronborg 1991) ein zweiter, als jungbaltischer bekannter Vorstoß. Er erreichte Dänemark hauptsächlich aus südöstlichen Richtungen und erstreckte sich über den gesamten östlichen Teil Dänemarks ebenfalls bis nach Jütland, wo er an der ostjütischen Eisrandlinie zum Stillstand kam (Abb. 1). Auf Fünen ist dieser Vorstoß im Bereich der Sand- und Kiesgruben durch einen 1 bis $4 \mathrm{~m}$ mächtigen Till an der Oberfläche der flachen Moränenlandschaft dokumentiert. Er folgt meist direkt auf den Till des Hauptvorstoßes und ist in seinem unteren Bereich z.T. durch ein schwach ausgebildetes ostsüdost-orientiertes Gefüge geprägt. Im oberen Abschnitt ist eine SSE-NNW Ausrichtung der Geschiebe vorherrschend (JørGENSEN \& Piotrowski 2003). Gegenüber dem älteren Till ist er auch mit Hilfe seines Geschiebeinhalts aus dem Bereich der Ostsee und seinem höheren Tongehalt abzugrenzen. Während der Weichsel-Hauptvorstoß durch ein relativ langsames, gleichmässiges Aufwachsen gekennzeichnet war, stieß der jungbaltische Gletscher in Form eines Eisstroms schnell vor (STEPHAN 2001, Jørgensen \& Piotrowski 2003). Seine Dynamik ist vergleichbar mit den heutigen Eisströmen der Westantarktis, die sich um einige Grössenordnungen schneller als das umliegende Eis bewegen (BentLey 1987).

\section{Methodik}

Die Aufnahme und Darstellung der Profile erfolgte unter besonderer Berücksichtigung der Lithologie, der Sedimentstruktur und der glazialtektonischen Deformation. Die Ermittlung der Stauchungsrichtung erfolgte mit Hilfe stereographischer Darstellung im Schmidtschen Netz (untere Hemisphäre).

In den beiden Tills wurde die Orientierung von Geschiebelangachsen untersucht (ANDREws
1971, Bennett et al. 1999), um Hinweise auf die Bewegungsrichtung der Gletscher zu erhalten. Ergänzend liefern die Ergebnisse von mehreren Feinkiesanalysen der 2-4 mm Kornfraktion (Kronborg 1995) Aufschlüsse über die Herkunft der Gletscher und ermöglichen damit eine lithostratigraphische Gliederung und Parallelisierung der Tills. Das ebenfalls untersuchte Korngrößenspektrum (DIN 1996) bietet ein zusätzliches Unterscheidungskriterium.

\section{Lokalität Ibjerg}

An der östlichen Abbauwand der Kiesgrube sind in einem bis zu 3,5 m mächtigen Profil hauptsächlich glazifluviatile Sande und Feinkiese aufgeschlossen (Abb. 3). Darüber war ursprünglich im gesamten Profil ein etwa 1 bis 1,5 $\mathrm{m}$ mächtiger Till diskordant abgelagert. Dieser wurde im Vorfeld des Sandabbaus größtenteils entfernt bzw. aufgeschoben und tritt heute in situ nur noch in wenigen Abschnitten und nicht mehr in voller Mächtigkeit auf.

\section{Lithologische Einheiten}

Während der untere Teil des Profils im Wesentlichen aus kiesführenden Grobsanden oder Feinkiesen besteht, verringern sich die Korngrößen in den darüber liegenden Profilbereichen bis hin zu schluffigen Feinsanden. An Sedimentstrukturen tritt überwiegend horizontale Schichtung auf. Innerhalb einzelner Bänke ist eine Gradierung zu beobachten.

Der am Top des Profils aufgeschlossene Till ist braun oder graubraun gefärbt und bildet in der Regel eine relativ feste, massive Einheit. In Korngrößenanalysen zeigt er ein ausgeprägtes Maximum im Feinsand und mittlere Korngrößen zwischen Mittel- und Grobschluff. Daneben ist zwischen 16 und 19\% Ton enthalten. Abweichungen von diesem Kornspektrum weist besonders die Tillbasis auf, wo häufig Sandbänder in den Till eingearbeitet sind. Die an zwei Stellen eingemessenen Ge- 

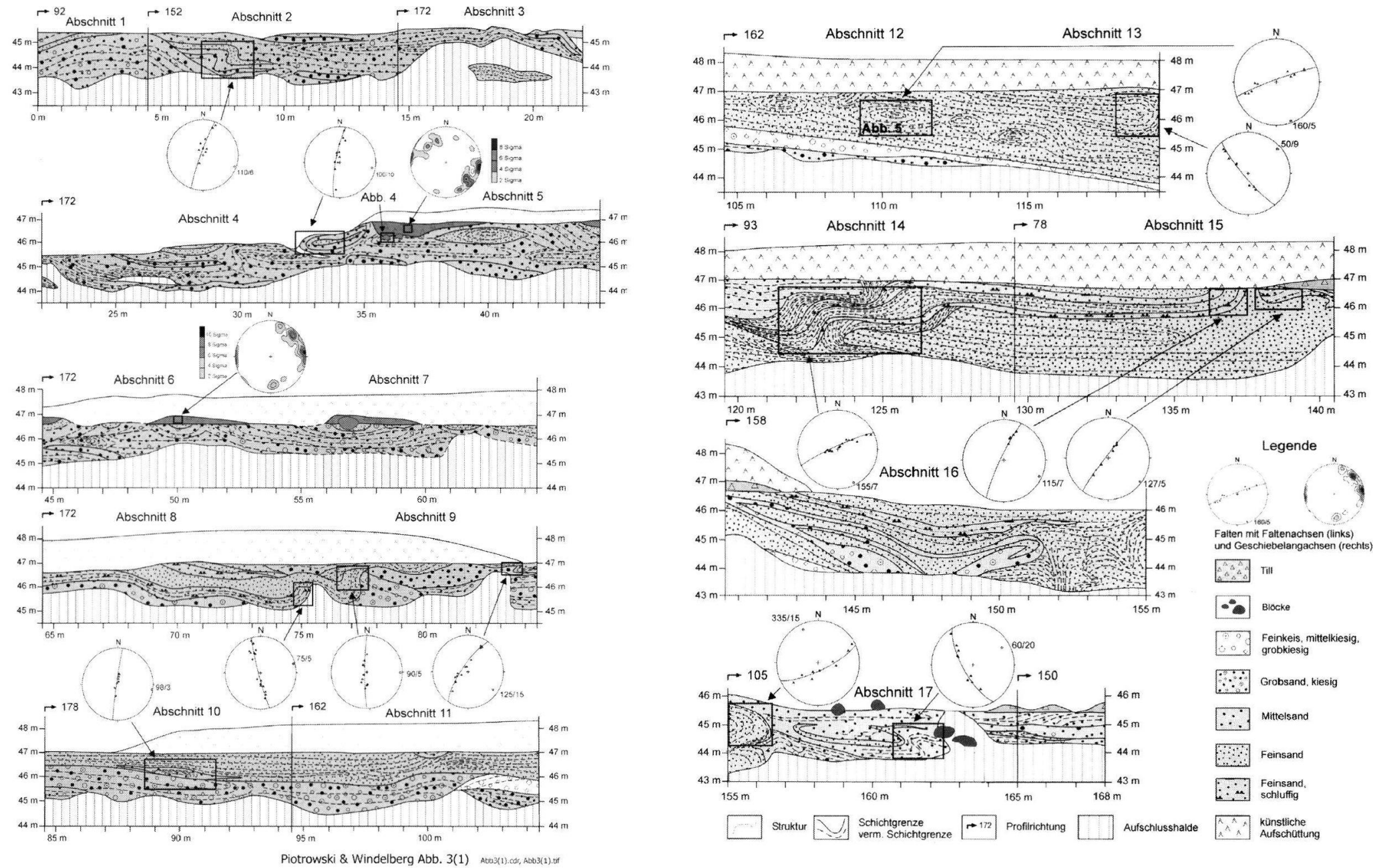

Abb. 3: Profil Ibjerg. Geschiebelangachsen dargestellt auf der unteren Hemisphäre des Schmidtschen Netzes. Die Lage des Profils zeigt Abb. 2.

Fig. 3: Section Ibjerg. Till fabric given on the lower hemisphere of the Schmidt projection. Section location in Fig. 2. 
schiebelangachsen weisen deutlich ausgeprägte aber verschiedene Maxima auf, nämlich NE (bevorzugte Fallrichtung) und SE. An der TillBasis treten größere Geschiebe oder Blöcke mit bis zu $1 \mathrm{~m}$ Durchmesser auf, welche teilweise in die darunter lagernden glazifluviatilen Sedimente eingepflügt sind und somit deutlich langgestreckte Erosionsrillen bilden (ploughing marks; Abb. 4), die die Eisvorstoßrichtung anzeigen (Ehlers \& Stephan 1979, Clark \& Hansel 1989, Menzies \& Shilts 2002). Sie sind, genauso wie die Geschiebelangachsen im unmittelbar auflagernden Till im Abschnitt 5, SE-NW orientiert. Aufgrund dieser Erosionsstrukturen, des scharfen basalen Kontaktes, der bimodalen Korngrößenverteilung (HALDORSEN 1981) sowie des Einregelungsverhaltens der Geschiebelangachsen (Dowdeswell et al. 1985) wird dieser Till als Setztill gedeutet (Dreimanis 1988, Piotrowski 1992).

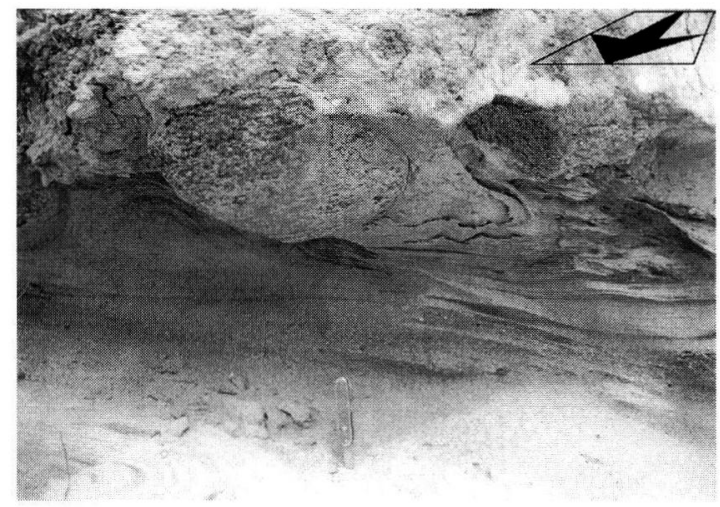

Abb. 4: Eine Erosionsrille im Schatten eines Gesteins an der Basis des jungbaltischen Tills in Ibjerg. Die Eisvorstoßrichtung (aus SE) ist gekennzeichnet mit dem Pfeil. Blickrichtung nach Osten, Lage der Aufnahme in Abb. 3.

Fig. 4: A plouging mark behind a boulder at the base of the Young Baltic till in Ibjerg. Arrow indicates the ice movement direction (from SE). View to E, photograph location in Fig. 3.

\section{Glazialtektonik}

Innerhalb der Sande wurden insgesamt 13 Falten sowie zahlreiche deformierte Flächen einge- messen. Aus der Konstruktion der Faltenachsen geht hervor, dass die Deformation hauptsächlich aus drei verschiedenen Richtungen erfolgte. Die intensivste Verformung erfuhren die Sedimente durch Eisschub aus $\mathrm{N}$ bis NE, welcher zur Bildung von etwas mehr als der Hälfte der eingemessenen Faltenstrukturen führte. Daneben wurden an drei Falten Richtungen gemessen, die auf einen Vorstoß aus SE bis SSE schließen lassen. Eine dritte Richtung, die ebenfalls anhand von drei Falten dokumentiert ist, entstand als Folge eines Vorstoßes aus ENE. Bemerkenswert ist, dass keine der gemessenen Richtungen in einem horizontal oder vertikal begrenzten Bereich innerhalb des Profils auftritt, sondern alle in unterschiedlichen Profilteilen und Profilhöhen ausgebildet sind.

Als Ursache für die unterschiedlichen Deformationsrichtungen werden im Folgenden drei Möglichkeiten diskutiert, die auch in Kombinationen für die Ausbildung dieser Richtungen verantwortlich sein können. Die naheliegendste Erklärung ist, dass die einzelnen Deformationsrichtungen jeweils durch verschiedene Eisvorstöße entstanden, die Fünen aus unterschiedlichen Richtungen erreichten.

Die zweite Erklärungsmöglichkeit beruht darauf, dass verschiedene Richtungen von Deformationsstrukturen infolge einer starken Gliederung des Eisrandes entstehen können. Dabei bilden sich Eisloben, welche den Gletscherrand in mehrere halbkreisförmige, zungenartige Bereiche unterteilen. Da sich ein Gletscher annährend senkrecht zu seinem Rand ausbreitet, können dadurch in jeder Richtung Abweichungen bis zu $90^{\circ}$ von der allgemeinen Vorstoßrichtung des Gletschers auftreten (STEPHAn 1985, SmeD 1997). Die drei im Profil gemessenen Deformationsrichtungen könnten auf diese Weise während einer einzigen Vereisungsphase entstanden sein. Dies ist jedoch aufgrund der Tatsache, dass keine der genannten Richtungen nur in einem definierbaren Teil des Profils auftritt, unwahrscheinlich. Möglich ist jedoch, dass die gemessenen Richtungen nur die Vorstoßrichtung am 
Lobenrand, nicht aber die Bewegungsrichtung des Gletschers insgesamt wiedergeben.

Eine dritte Möglichkeit der Entstehung der unterschiedlichen Deformationsrichtungen wird durch das Auftreten von Zungenfalten (sheath folds) gegeben (vgl. Abb. 5). Sie entstehen meist durch heterogene einfache Scherung unter sehr hohen Scherbelastungen. Die Krümmung der Faltenachse erfolgt im Umfeld progressiver Scherung, indem die Faltenachsen seitlich in die Scherrichtung hinein rotieren (VAN DER WATEREN 2002, Burg 2001). Unter der Annahme, dass die eingemessenen Strukturen verschiedene Bereiche und Entwicklungsstadien von Zungenfalten darstellen, wäre die Abweichung der Streichrichtungen somit erklärbar. Da sich jedoch nur ein geringerer Teil der Falten in Ibjerg eindeutig als Zungenfalten identifizieren lässt, kann das Auftreten der unterschiedlichen Deformationsrichtungen nicht allein im $\mathrm{Zu}$ sammenhang mit den Zungenfalten erklärt werden.

Weiterhin stellt sich die Frage, wie der jüngste Eisvorstoß das Profil in Teilbereichen bis zur

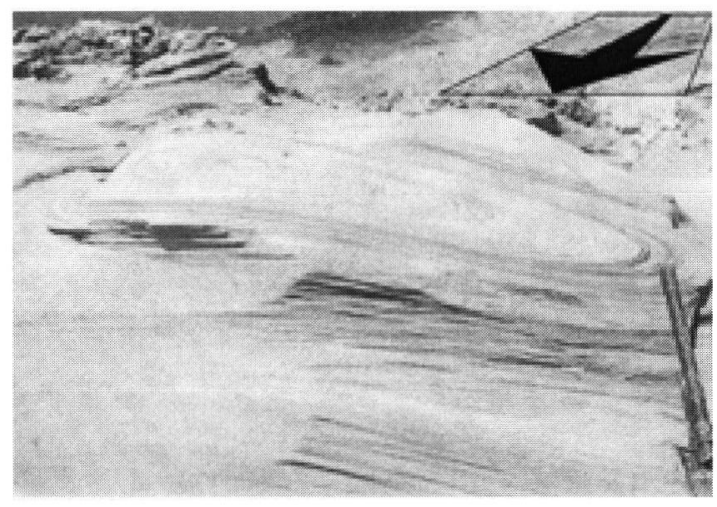

Abb. 5: Eine Zungenfalte im Schmelzwassersand unter dem jungbaltischen Till in Ibjerg. Die Eisvorstoßrichtung (aus SE) ist gekennzeichnet mit dem Pfeil. Blickrichtung nach Osten, Lage der Aufnahme in Abb. 3.

Fig. 5: A sheath fold in meltwater sand under the Young Baltic till in Ibjerg. Arrow indicates the ice movement direction (from SE). View to E, photograph location in Fig. 3.
Profilbasis überprägen konnte, während in anderen Teilen ältere Deformationsrichtungen erhalten blieben. Generell tritt subglaziale Deformation bei einer verminderten Scherfestigkeit des Untergrundes auf. Sie kann z.B. durch einen erhöhten Porenwasserdruck bedingt sein, welcher subglazial als Folge eines eingeschränkten Schmelzwasserabflusses im Sediment auftritt (Menzies 1989, Piotrowski \& Kraus 1997). Da Sedimente mit geringer Durchlässigkeit einen hohen Porenwasserdruck länger beibehalten als Sedimente mit hoher Durchlässigkeit, werden erstere leichter deformiert (Van der Wateren 2002). Möglicherweise sind die voneinander abweichenden Deformationsrichtungen der kartierten Sande eine Folge ihrer unterschiedlichen Durchlässigkeit, welche sich in den deutlich voneinander abweichenden Korngrößenverteilungen zeigt. So könnten Deformationen an verschiedenen Stellen in der Sedimentfolge zu unterschiedlichen Zeiten entstehen, erhalten bleiben oder auch später überprägt werden, was zu dem unsystematischen Erscheinungsbild der Stauchungsrichtungen führte.

Eine weitere Erklärungsmöglichkeit für das Auftreten der drei Deformationsrichtungen nebeneinander ist die hydraulische Anhebung des Gletschers vom subglazialen Sediment in jeweils einzelnen Teilbereichen des Profils durch einen hohen subglazialen Wasserdruck (PIOTROWSKI \& Tulaczyk 1999, Munro-Stasiuk 2000, FisHER \& TAYLOR 2003). Ein dünner Wasserfilm zwischen dem Eis und den unterlagernden Sedimenten schwächt örtlich die Übertragung der Scherspannungen auf den Untergrund $a b$, so dass ältere Deformationsstrukturen nicht mehr überprägt werden.

\section{Lokalität Birkum}

In dem aufgenommenen, zwischen 4 und $5 \mathrm{~m}$ mächtigen Profil (Abb. 6) lassen sich aufgrund lithologischer Kriterien, teilweise mit Hilfe von 
Kornverteilungs- und Feinkiesanalysen, fünf Einheiten unterscheiden. Von diesen treten wegen intensiver Deformation und Überschiebung eines Großteils der Abfolge einige Einheiten nicht in allen Abschnitten auf.

\section{Lithologische Einheiten}

Im gesamten Profil sind nahe der Profilbasis bis zu $2 \mathrm{~m}$ mächtige braune, teils feinsandig teils tonig ausgebildete Schluffe und seltener schluffige Tone aufgeschlossen, in welche zahlreiche Dropstones eingelagert sind. Die Schluffe sind sehr kompakt und zeigen meist eine deutliche Lamination, bestehend aus einzelnen zwischen $5 \mathrm{~mm}$ und $3 \mathrm{~cm}$ mächtigen Lamina. Zum Hangenden werden sie durch einige Millimeter bis mehrere Zentimeter mächtige Sandlagen unterbrochen. Letztere werden nach oben hin mächtiger und gröber. Sie bilden den sich oberhalb der Schluffe anschließenden Sandkörper.

Die Sande sind sehr heterogen aus geschichteten Fein-, Mittel- und Grobsanden zusammengesetzt und weisen aufgrund starker glazialtektonischer Beanspruchung in Form von Faltung, Überkippung und Verschleppung im Profil Mächtigkeiten zwischen einigen Zentimetern und mehr als einem Meter auf. Je nach Korngröße sind die einzelnen Lagen braun, gelbbraun oder weiß gefärbt.

Über den Sanden treten in einigen Abschnitten hellgraue und weiße grobsandige Kiese mit scharfem Kontakt zu den Sanden auf. Sie besitzen ein breites Korngrößenspektrum von Feinsand bis hin zu Blöcken mit Durchmessern von $25 \mathrm{~cm}$. Auch die Kiese weisen eine starke Deformation auf, da sie wie die Sande sowohl gefaltet als auch z.T. überkippt sind. Dies führt im Abschnitt 6 zu einer knapp $3 \mathrm{~m}$ mächtigen Kiesstruktur, während die Kieslagen sonst zwischen 10 und $25 \mathrm{~cm}$ mächtig sind.

Abgeschlossen wird die Profilfolge von zwei Tills. Der untere der beiden ist braun bis graubraun und abgesehen von einigen weichen Sandlinsen und -schlieren relativ hart. Besonders im oberen Bereich ist die Tillstruktur durch ein vermehrtes Auftreten von Geschieben in Grobkies- bis Blockgröße gekennzeichnet. Insgesamt ist die Kornzusammensetzung des Tills durch eine mittlere Korngröße im Grobschluffbereich, einen hohen Feinsandgehalt und Tonanteile zwischen 9 und 13,5\% geprägt.

Optisch ist der darüber lagernde obere Till dem unteren sehr ähnlich. Eine Abgrenzung ist vor allem aufgrund seiner, im Gegensatz zu den übrigen aufgeschlossenen Einheiten, ungestörten Lagerung möglich. Im Vergleich zum unteren Till ist er zudem etwas feinkörniger und dementsprechend fester. Aufgrund der nur geringen Durchsetzung mit Sandschlieren und dem mit 14,5 und $16 \%$ relativ hohen Tongehalt ist er massiger als der untere Till. Seine Mächtigkeit ist abhängig von der Morphologie älterer Sedimente und liegt zwischen 0,5 und $1,5 \mathrm{~m}$. Zwei Geschiebelangachsen-Messungen im oberen Till zeigen generell eine Fallrichtung nach ESE an, wobei die weniger deutliche, bimodale Verteilung der Fallrichtungen im Abschnitt 4 auf glazialtektonische Überprägung zurückgeführt wird.

Während eine Bestimmung der Tillfazies im unteren Till aufgrund der Deformation mit den angewendeten Methoden nicht möglich ist, kann der obere Till relativ eindeutig als Setztill identifiziert werden. Darauf deutet seine hohe Festigkeit, die insgesamt massige Struktur, die bimodale Korngrößenverteilung, und die aus der Geschiebeeinregelung berechneten Eigenwerte (MARK 1973) hin. Sie zeigen das für Setztills typische deutliche Einregelungsverhalten der Geschiebelangachsen parallel zur Eisbewegungsrichtung.

\subsection{Glazialtektonik}

Als dominierende glazialtektonische Deformationen treten im Profil Birkum drei Überschiebungsstrukturen auf, welche in den Abschnitten 1, 3 und 6 mit der Faltung der Schluffe, Sande und Kiese beginnen. Diese Falten sind 

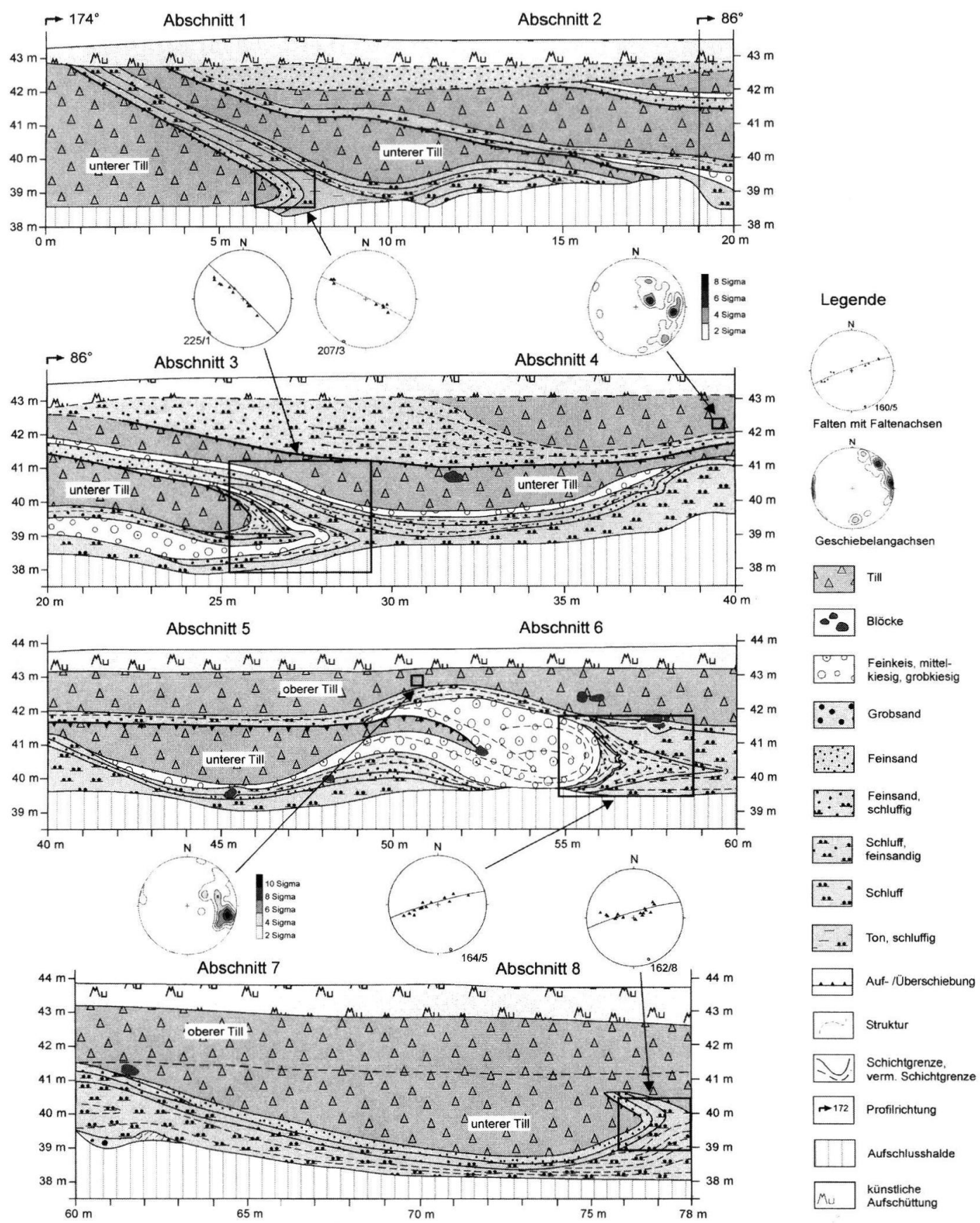

Abb. 6: Profil Birkum. Geschiebelangachsen, dargestellt auf der unteren Hemisphäre des Schmidtschen Netzes. Die Lage des Profils zeigt Abb. 2.

Fig. 6: Section Birkum. Till fabric given on the lower hemisphere of the Schmidt projection. Section position in Fig. 2. 
überkippt und im Profil bis zu $40 \mathrm{~m}$ weit über den unteren Till überschoben. Dabei wurden vor allem die Sande, aber teilweise auch die Schluffe und die Kiese entlang einer Überschiebungsbahn an der Oberfläche des Tills lateral gegenüber diesem bewegt. Erklärbar ist die Bewegung der relativ grobkörnigen Sedimentfolge entlang der Überschiebungsfläche nur durch vollständige Wassersättigung und einen hohen Porenwasserdruck während der Deformationsphase. Die innerhalb der Falte größtenteils erhaltenen Sedimentstrukturen wie die Schichtung der Sande und Kiese und die Lamination der Schluffe zeugen von einer heterogenen Verteilung der Deformation im Sediment, wobei die größte Verformung im feinkörnigen Sediment und an Schichtgrenzen standfand.

Die anhand der drei großen Überschiebungsbahnen und der dazugehörigen Faltenstrukturen ermittelten Lagerungswerte zeigen zwei Deformationsrichtungen. Dabei handelt es sich zum einen um die in den Profilabschnitten 1 bis 3 auftretende NE-SW streichende Richtung der Faltenachsen, zum anderen um die in den Profilabschnitten 4 bis 8 zusätzlich auftretende SSE-NNW Streichrichtung. Basierend auf diesen Daten wird von folgender Genese ausgegangen: Nach der Ablagerung der Sedimente mit Ausnahme des oberen Tills deformierte ein aus NE bis ENE heranrückender Gletscher den östlichen Teil des Profils mit den Profilabschnitten 4 bis 8. Dabei entstanden die in SSE-NNW streichenden Falten. $\mathrm{Ob}$ auch die Profilabschnitte 1 bis 3 durch diesen Vorstoß deformiert wurden, ist aus den vorhandenen Strukturen in diesem Bereich nicht ersichtlich. Nach dem Abschmelzen des Nordost-Gletschers kam es durch einen Gletscher aus SE zu einer erneuten Deformation, die in allen Profilteilen durch NE-SW streichende Faltenstrukturen nachweisbar ist. Während dieses Eisvorstoßes wurde der obere Till abgelagert, wie aus den größtenteils südöstlichen Einfallsrichtungen der gemessenen Geschiebelangachsen hervorgeht.

\section{Stratigraphie}

Da mittels der durchgeführten Analysen die stratigraphische Stellung der verschiedenen Sande, Schluffe und Kiese weder in Birkum noch in Ibjerg eindeutig geklärt werden konnte, sind über eine Korrelation dieser Einheiten nur Vermutungen möglich. JøRGENSEN (1996) zufolge gehören die in Ibjerg aufgeschlossenen Sande zu einer im Bereich der Kiesgruben weit verbreiteten mächtigen Schmelzwassereinheit. Diese lässt sich vermutlich mit den Sanden korrelieren, welche in Birkum direkt unterhalb der Schluffe anstehen, im Profil aber nicht aufgeschlossen sind.

Für den unteren Till in der Lokalität Birkum wird eine Entstehung während des Hauptvorstoßes der Weichselzeit angenommen. Dafür sprechen der hohe Flintgehalt (durchschnittlich 34\%) im Feinkiesanteil (Abb. 7) und relativ geringe Quarz- (10\%) und Sedimentgehalte (5\%), welche für den Till des weichselzeitlichen Hauptvorstoßes typisch sind (KRONBORG 1995). Obwohl der Anteil der paläozoischen Kalke (23\%) höher liegt als die durchschnittlichen 10 bis $15 \%$, ist er nach Houmark-Nielsen (1987) für den Till im zentralen Teil Dänemarks nicht ungewöhnlich. Nach Jørgensen \& PiOTROwSKI (2003) ist auch der geringe Tonanteil (9 bis 13,5\%) ein charakteristisches Merkmal dieses Tills.

Im Gegensatz zum unteren Till zeigen die Ergebnisse im oberen Till Vorstoßrichtungen aus $\mathrm{E}$ und SE, sodass von einer Ablagerung des jungbaltischen Vorstoßes auszugehen ist. Diese Einschätzung wird durch den vergleichsweise hohen Tonanteil (14,5 bis 16\%) und die Ergebnisse der Feinkiesanalyse gestützt, welche geringfügig erhöhte Anteile von Gesteinen der Ostsee, vornehmlich paläozoischen Kalken und Sedimentgesteinen, aufweist.

Deutlicher als der obere Till in der Lokalität Birkum zeigt der Till am Ibjerg Kennzeichen des jungbaltischen Vorstosses. Neben den hohen Tonanteilen von 16 und 19\% in den beiden 


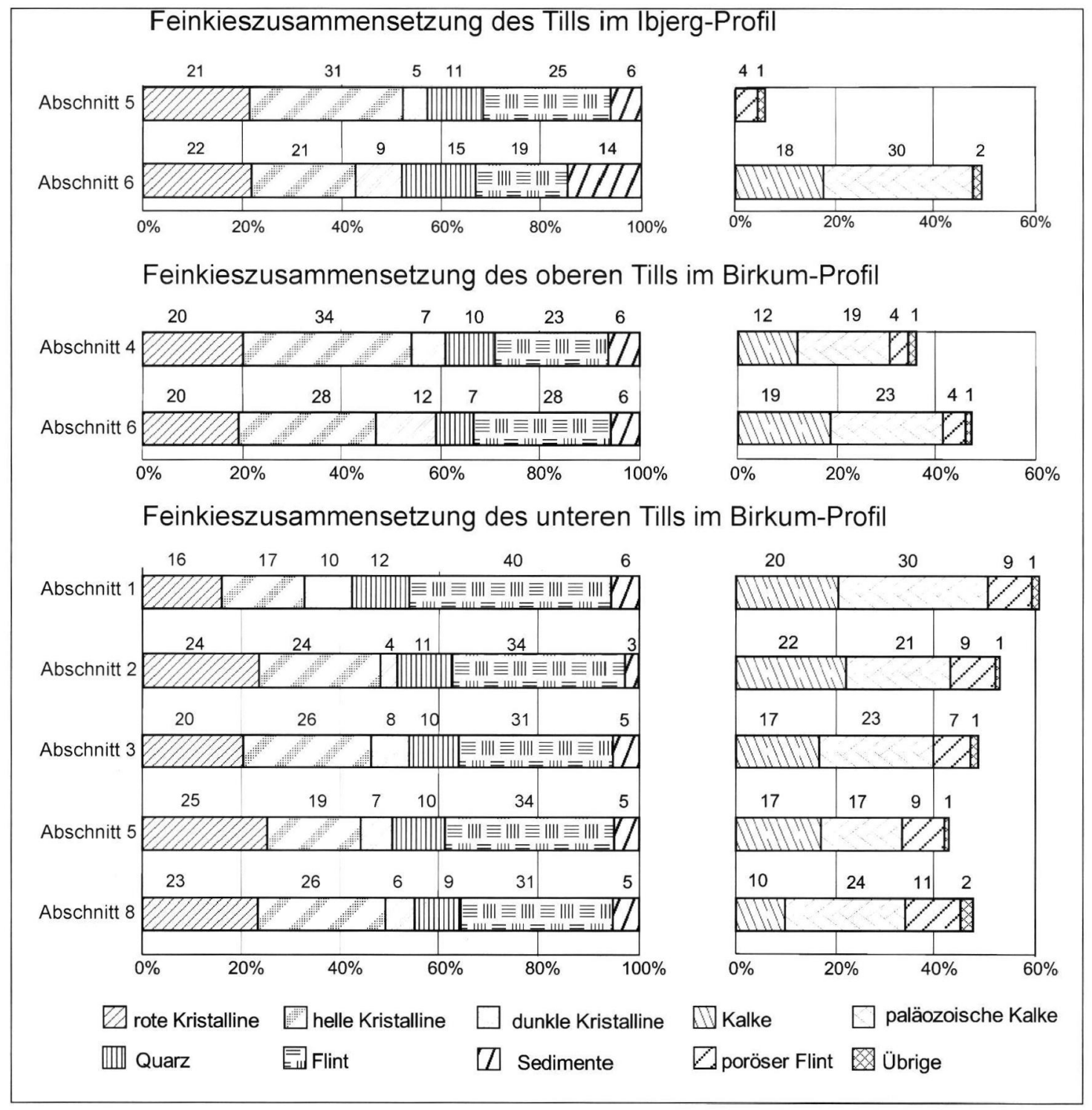

Abb. 7: Feinkieszusammensetzung der Tills in Ibjerg und Birkum.

Fig. 7: Fine-gravel composition of tills in Ibjerg and Birkum.

analysierten Proben bietet die Orientierung von drei eingemessen Pflugstrukturen (Streich- und Fallrichtungen zwischen $120^{\circ}$ und $123^{\circ}$ ) und der in Abb. 7 dargestellte Feinkiesinhalt mit einem paläozoischen Kalkanteil von 30\% und einem Sedimentgehalt von durchschnittlich $10 \%$ deutliche Anhaltspunkte für den jüngsten weichselzeitlichen Vorstoß. Aufgrund der Position des Tills direkt oberhalb der wesent- lich älteren glazifluviatilen Sande, ist davon auszugehen, dass der Till des weichselzeitlichen Hauptvorstoßes hier entweder nicht abgelagert oder während des nachfolgenden jungbaltischen Vorstoßes vollständig erodiert wurde. Die TillLinse im Abschnitt 6 mit der NE-Einregelung der Geschiebelangachsen könnte zwar ein Rest des Weichsel-Hauptvorstoß-Tills sein, es ist aber wahrscheinlicher, dass diese Einregelung 
während des jungbaltischen Vorstoßes unter kompressiven Eisfliessbedingungen als b-Einregelung entstand (vgl. Boulton 1971).

\section{Zusammenfassende Diskussion}

Die beiden analysierten Profile zeigen deutliche Unterschiede in der Wirkungsweise und dem Erscheinungsbild der Deformation. In Folge der geringen Permeabilität der feinkörnigen Sedimente und eines hohen Porenwasserdrucks sind in der Lokalität Birkum ohne signifikante Veränderung der inneren Sedimentstrukturen mehrere, sich zum Teil überlagernde große Überschiebungsstrukturen entstanden. Sie lassen sich klar den beiden weichselzeitlichen Eisvorstößen, dem aus NE kommenden Hauptvorstoß und dem aus SE kommenden jungbaltischen Vorstoß zuordnen. Trotz unterschiedlicher Eisbewegungsdynamik (ein relativ langsames, stationäres Eisfliessen gegenüber einem schnellen Eisstrom), haben beide Vorstöße ähnliche Deformationsstrukturen hinterlassen. Dies deutet darauf hin, dass der Deformationsstil mehr durch die geologischen als glaziologischen Parameter gesteuert war.

Im Unterschied dazu führten Scherspannungen unter denselben Eisvorstößen in der Lokalität Ibjerg zu intensiven Faltungen der grobkörnigen Sedimente unter Veränderung oder völliger Zerstörung der internen Sedimentstruktur. Es wird dabei von einer selektiven Deformation ausgegangen, die zu horizontal und vertikal nicht zusammenhängenden Stauchungsrichtungen führte. Dieses Deformationsmuster wird auf unterschiedliche Scherfestigkeiten zurückgeführt, die wiederum durch Unterschiede in den Korngrößen und damit verschiedene Porenwasserdrücke hervorgerufen waren. $\mathrm{Da}$ auch hier, trotz unterschiedlicher Dynamik der beiden Eisvorstöße grundsätzlich ein Stil der glazialtektonischen Deformationen überwiegt, wird den mechanischen Eigenschaften des Untergrundes eine grössere Rolle für die
Schaffung eines bestimmten Deformationsstil als den glaziologischen Prozessen, wie z.B. die Gletscherbewegungsgeschwindigkeit oder das Eisprofil, beigemessen.

Die Feststellung der tiefgreifenden Sedimentverformung in beiden Lokalitäten, insbesondere der für eine hohe Scherbeanspruchung diagnostischen Zungenfalten (sheath folds) in Ibjerg, liefert einen wichtigen Hinweis auf den Bewegungsmechanismus des ehemaligen Eisstroms der skandinavischen Eiskappe. Es ist bekannt, dass moderne Eisströme ihre hohe Geschwindigkeiten durch das Gleiten auf einem dünnen Wasserfilm (ENGELHARDT \& Камв 1998), durch intensive Verformung des weichen Untergrundes (ALLEY et al. 1986) oder durch Kombination beider Mechanismen (TuLACZYK et al. 2001) erreichen. Viel unsicherer ist die Rekonstruktion der Bewegungsmechanismen pleistozäner Eisströme, die nur anhand geologischer und geomorphologischer Hinweise gemacht werden kann (StOKes \& Clark 2003). Bei der Analyse des jungbaltischen Eisstroms auf Fünen schlugen Jørgensen \& Piotrowski (2003) vor, dass eine Kombination der Untergrundverformung und des basalen Gleitens Ursache für die schnelle Gletscherbewegung war. Die Ergebnisse der vorliegenden Arbeit bestätigen eindeutig die Untergrundverformung und durch den indirekt abgeleiteten starken Wasserdruck an der Gletscherbasis legen sie auch das verstärkte basale Gleiten nahe. Diese beiden Mechanismen dürften in Zeit und Raum varriert haben, sodass die Grenzfläche Gletscher/Untergund als ein instationäres Mosaik des gekoppelten und aufschwebenden Eises angesehen werden kann (Piotrowski \& Kraus 1997, KNight 2002).

\section{Danksagung}

Für die Gewährung des Zuganges zum Kiesabbaugebiet sei den Firmen NCC und NS Nymølle Stenindustrier A/S aus Oddense gedankt. 
Für die Einführung in die Geologie Fünens, anregende Diskussionen und zahlreiche Tips bedanken wir uns bei den Herren Flemming Jørgensen, Vejle Amt, und Gunnar Larsen, Fyns Amt. Gedankt sei auch den Gutachtern und dem Schriftleiter für wertvolle Kritik und Anregungen.

\section{Schriftenverzeichnis}

Aber, J., Croot, D. G. \& Fenton, M. M. (1989): Glaciotectonic Landforms and Structures.- 201 S.; Dordrecht (Kluwer Academic Publishers).

Alley, R. B., Blankenship, D. D., Bentley, C. R. \& RoOney, S. T. (1986): Deformation of till beneath Ice Stream B, West Antarctica.Nature, 322: 57-59; London.

Andrews, J. T. (1971): Techniques of Till Fabric Analysis. - Technical Bulletin, British Geomorphological Research Group, 6: 1-43.

Bennett, M. R., Walder, R. I., Glasser, N. F., Hambrey, M. J. \& Huddard, D. (1999): Glacigenic clast fabrics: genetic fingerprint or wishful thinking?- Journal of Quaternary Science, 14 (2): 125-135; Chichester.

Bentley, C. R. (1987): Antarctic ice streams: a review.- Journal of Geophysical Research, 92 (B9): 8843-8858.

Boulton, G.S. (1971): Till genesis and fabric in Svalbard, Spitsbergen.- In: Goldthwart, R.P. [Hrsg.]: Till: a symposium: 41-72; Columbus (Ohio State University Press).

BurG, J.-P. (2001): Einführung in die Strukturgeologie.- http://e-collection.ethbib.eth $\mathrm{z} . \mathrm{ch} /$ show?type $=\mathrm{lehr} \& \mathrm{nr}=11 \quad$ Geologisches Institut ETH Zürich).

Clark, P. U. \& Hansel, A. K. (1989): Clast ploughing, lodgement and glacier sliding over a soft glacier bed.- Boreas, 18: 201-207; Oslo.

DIN Deutsches Institut für Normung E. V. (1996): DIN 18123 Bestimmung der Korngrößenverteilung.- $12 \mathrm{~S}$.; Berlin (Beuth).
Dowdeswell, J. A., Hambrey, M. J. \& Wu, R. (1985): A comparison of clast fabric and shape in Late Precambrian and modern glacigenic sediments.- Journal of Sedimentary Petrology, 55 (5): 691-704; Tulsa.

Dreimanis, A. (1988): Tills: their genetic terminology and classification.- In: GolDThwait R. P \& Matsch, C. L. [Hrsg.]: Genetic Classification of Glacigenic Deposits: 17-83; Rotterdam (Balkema).

Ehlers, J. \& Stephan, H.-J. (1979): Forms at the base of till strata as indicators of ice movement.- Journal of Glaciology, 22: 345356; Cambridge.

Engelhardt, H. F. \& Kamb, B. (1998): Basal sliding of Ice Stream B, West Antarctica.Journal of Glaciology, 44: 223-230; Cambridge.

Fisher, T. G. \& TAylor, L. D. (2003): Sedimentary and stratigraphic evidence for subglacial flooding, south-central Michigan, USA.- Quaternary International, 90: 87115; Oxford.

Geoditisk Institut (1987): Danmark 1: 25000, 1312 I NV Odense. Kopenhagen (Geodætisk Institut).

Haldorsen, S. (1981): Grain-size distribution of subglacial till and its relation to subglacial crushing and abrasion.- Boreas, 10: 91-105; Oslo.

Houmark-Nielsen, M. (1981): Glacialstratigrafi i Danmark øst for Hovedopsholdslinien.- Dansk Geologisk Forening, Årsskrift for 1980: 61-76; Kopenhagen.

Houmark-Nielsen, M. (1987): Pleistocene stratigraphy and glacial history of the central part of Denmark.- Bulletin of the Geological Society of Denmark, 36: 1-189; Kopenhagen.

Houmark-Nielsen, M. (1999): A lithostratigraphy of Weichselian glacial and interstadial deposits in Denmark.- Bulletin of the Geological Society of Denmark, 46: 101114; Kopenhagen.

Jørgensen, F. V. (1996): En undersøgelse af 
østfyns glacialstratigrafi og landskabsformer.- 293 S. Dipl. Arb., Univ. Aarhus, Aarhus (unveröff.).

Jørgensen, F. V. \& Piotrowski, J. A. (2003):

Signature of the Baltic Ice Stream on Funen

Island, Denmark during the Weichselian glaciation.- Boreas, 32 (1): 242-255; Oslo.

Kjar, K., Houmark-Nielsen, M. \& Richardt, N. (2003): Ice-flow patterns and dispersal of erratics at the southwestern margin of the last Scandinavian Ice Sheet: signature of palaeo-ice streams.- Boreas, 32 (1): 130-148; Oslo.

KNIGHT, J. (2002): Glacial sedimentary evidence supporting stick-slip basal ice flow.Quaternary Science Reviews, 21: 975-983; Amsterdam.

Kronborg, C. (1995): Kvartæret.- In: Nielsen, O.B. [Hrsg.]: Aarhus Geokompendier Nr. 1, Denmarks geologi fra Kridt til i dag: 271290; Aarhus (Geologisk Institut).

Madsen, V. (1902): Beskrivelse til Geologisk Kort over Danmark. Kortbladet Nyborg. Danmarks Geologiske Undersøgelse I (9), 182 S; Kopenhagen.

Mark, D. M. (1973): Analysis of axial orientation data, including till fabrics.- Geological Society of America Bulletin, 84: 13691374.

Menzies, J. (1989): Asubglacial hydraulic conditions and their possible impact upon subglacial bed deformation.- Sedimentary Geology, 62: 125-150; Amsterdam.

Menzies, J. \& W. W. Shilts (2002): Subglacial environments.- In: MenzIEs, J. [Hrsg.]: Modern \& past glacial environments: 183-278; Oxford (Butterworth Heinemann).

Milthers, V. (1928): Glacialgeologiske Retningslinier i Odenseegnen.- Meddelelser fra Dansk Geologisk Forening, 7: 179-200; Kopenhagen.

Milthers, K. (1942): Ledeblokke og Landskabsformer i Danmark. Danmarks Geologiske Undersøgelse, II (69); Kopenhagen.

Munro-Stasiuk, M. J. (2000): Rhythmic till se- dimentation: Evidence for repeated hydraulic lifting of a stagnant ice mass.- Journal of Sedimentary Research, 70: 94-106; Tulsa.

Petersen, K. S. \& Kronborg, C. (1991): Late Pleistocene history of the inland glaciation in Denmark.- In: Frenzel, B. [Hrsg.]: Klimageschichtliche Probleme der letzten 130 000 Jahre: 331-242; Mainz (Akademie der Wissenschaften und der Literatur).

Piotrowski, J. A. (1992): Was ist ein Till?- Die Geowissenschaften, 4: 100-108; Weinheim. Piotrowski, J. A. \& Kraus, A. M. (1997): Response of sediment to ice-sheet loading in northwestern Germany: effective stresses and glacier-bed stability.- Journal of Glaciology, 43: 495-502; Oxford.

Piotrowski, J. A. \& Tulaczyk, S. (1999): Subglacial conditions under the last ice sheet in northwest Germany: ice-bed separation and enhanced basal sliding?- Quaternary Science Reviews, 18: 737-751; Amsterdam.

Smed, P. (1962): Studier over den fynske øgruppes glaciale landskabsformer.- Meddelser fra Dansk Geologisk Forening, 15: 1-74; Kopenhagen.

Smed, P. (1978): Det fynske landskab. 51 S.; Brenderup (Geografforlaget).

SMED, P. (1997): De 949 skuresten i Lindø-dokkerne.- Geologisk Nyt, 3: 22-25; Aarhus.

Stephan, H.-J. (1985): Deformations striking parallel to glacier movement as a problem in reconstructing its direction.- Bulletin of the Geological Society of Denmark, 34: 47-53; Kopenhagen.

Stephan, H.-J. (2001): The Young Baltic advance in the western Baltic depression.- Geological Quarterly, 45 (4): 359-364Warschau.

Stokes, C. R. \& Clark, C. D. (2003): Laurentide ice streaming on the Canadian Shield: A conflict with the soft-bedded ice stream paradigm?- Geology, 31 (4): 347350; Boulder.

Tulaczyk, S. M., Scherer, R. P. \& Clark, C. D. (2001): A ploughing model for the origin of weak tills beneath ice streams: a qualitati- 
ve treatment.- Quaternary International, 86:

59-70; Oxford.

Van der Wateren, F. M. (2002): Processes of glaciotectonism.- In: J. Menzies [Hrsg.]: Modern \& past glacial environments: 417444; Oxford (Butterworth Heinemann). 